\title{
Development of a small baby unit to improve outcomes for the extremely premature infant
}

\author{
Omid Fathi ${ }^{1} \cdot$ Leif D. Nelin ${ }^{1,2} \cdot$ Edward G. Shepherd $\mathbb{C}^{1} \cdot$ Kristina M. Reber $\mathbb{C}^{1}$
}

Received: 29 June 2020 / Revised: 17 November 2020 / Accepted: 2 February 2021 / Published online: 12 March 2021

(c) The Author(s), under exclusive licence to Springer Nature America, Inc. 2021

\begin{abstract}
Survival and outcomes for extremely premature (EP) infants have improved and even infants born at 23 and 24 weeks that were previously considered non-viable are now routinely surviving. This review describes our particular institution's basis for and process of creating and sustaining a small baby program for a quaternary, referral-based neonatal intensive care unit. Through multi-disciplinary collaboration, small baby guidelines were developed that established uniform care and optimized evidence-based practice for the care of this unique patient population. A focus on parent-centered care while removing noxious stimuli for the patient has improved neurodevelopmental outcomes. Data collection, quality improvement, and ongoing research are incorporated in the small baby program to establish and sustain best practices and outcomes for the EP patient. Through the establishment of a small baby unit, we have improved survival, decreased short-term morbidities, and improved neurodevelopmental outcomes for the EP infant in our region.
\end{abstract}

\section{Introduction}

Neonatal intensive care has improved steadily over time as we have introduced new approaches like non-invasive ventilation in the delivery room, antenatal steroids, and the routine use of surfactant. These improvements have led to marked improvements in survival for all groups of patients and have particularly improved survival for both very preterm (VP, 27-32 weeks gestation at birth) and extremely preterm (extremely premature (EP), $<27$ weeks gestation at birth) infants. Indeed, infants born at 23 and 24 weeks of gestation, once considered non-viable, are now routinely surviving, and some institutions are demonstrating remarkable success even prior to 23 weeks [1]. Unfortunately, these gains in survival have not been universal nor equally distributed across centers and geographic areas, and these differences in outcomes are not explained by

Kristina M. Reber

Kris.Reber@nationwidechildrens.org

1 Small Baby Unit, Division of Neonatology at Nationwide Children's Hospital and Department of Pediatrics at The Ohio State University Wexner Medical Center, Columbus, OH, USA

2 Center for Perinatal Research, The Research Institute, Nationwide Children's Hospital, Columbus, OH, USA differences in patient characteristics. For example, Stoll et al. in 2010 published survival data from the NICHD centers that varied from less than 5\% to greater than $50 \%$ at 23 weeks gestation and from 20 to $100 \%$ at 24 weeks gestation, with no detectable differences in indicators of acuity or illness severity [2]. Other studies have demonstrated similarly variable outcomes with improved survival related to the intensity of effort by both the obstetric and neonatal teams [3]. In other words, those centers that uniformly and aggressively treated mothers in labor and resuscitated EP infants had better survival than those that took a more case-by-case approach. These differences in outcomes do not seem to be resolving with time, as recently published data have suggested that survival rates at 22 weeks of gestation range from $0 \%$ to greater than $60 \%$ again based largely on differences in approach by the obstetric and neonatal medical teams [4]. Clearly, these data suggest that best practices likely exist that could improve outcomes if applied uniformly across the population of EP infants.

Both in pediatric and adult medicine, creating uniformly standardized care guidelines clearly improves outcomes in a variety of disease states [5-7]. Such improvements are typically based on methodology focused on reducing unintended and/or unnecessary variability, reliably adhering to evidence-based practices, reducing impediments to proper care, creating a comprehensive and multidisciplinary 
team, and regularly recording, assessing, and evaluating outcomes. Based on these observations and principles, we and other institutions created specific programs to improve the outcomes of EP babies. The remainder of this manuscript will describe our particular basis for, and process of, creating small baby programs.

\section{Variability of outcomes in small babies}

The medical care required to support the premature infant at the threshold of viability is unique and differs from that required for the later preterm ( $>27$ weeks) infant or the fullterm infant. Yet, all of the various gestational age populations are cared for in the quaternary neonatal intensive care unit (NICU). The EP infant requires a unique focus from each multidisciplinary team member and recognition of the value of providing a different approach to the care of this vulnerable patient population [8].

Inconsistency of care among EP infants in our NICU was a concern prior to the development of our small baby guidelines (SBG). However, it was not unique to our NICU, as care for EP infants has varied across institutions around the world $[9,10]$. Contributing to this inconsistency of care of the EP infant is a lack of evidence in best practices for care and highly variable attitudes of care-givers related to the peri-viable infant [11]. At the time of the initial development of the small baby unit within the NICU at Nationwide Children's Hospital, there was little evidence on best clinical practices for the care of EP infants available. The lack of evidence led to highly variable practice approaches in the care of the EP infant and contributed to worse outcomes [12]. The variation in care was further enabled by the size of our unit, which is the only Level IV NICU in a 34county referral region in Southeastern Ohio. Furthermore, our Level IV NICU is an all referral unit without a labor and delivery suite. There are three separate physician practice groups who admit to the NICU (represented by more than 50 neonatologists), over 150 nurses, 25 respiratory therapists, and a large number of ancillary neonatal professionals including nutritionists, pharmacists, occupational and physical therapists, and social workers involved in the care of the NICU patients. The approach to the care of the EP infant was inconsistent and often exacerbated by caregivers frequently changing day-to-day due to rotating work schedules. A growing group of physicians and staff recognized the significant difference not only in the approaches to care for the EP infant but also in the attitudes of the attending physicians and staff related to the chances for survival and ethical considerations related to a perceived overly aggressive approach to the care of the EP infant. This led to "mixed messages" and inconsistent communication with families, which severely hampered family participation in care and their trust in their infants' caregivers. A small group of physicians and staff recognized that to improve outcomes and integrate families into care that a standard approach to the EP infant was absolutely necessary. They also recognized that in conjunction with this standard approach a data collection and monitoring system would need to be established to optimize the on-going care of the EP infants and their families. This situation was made even more complicated by the fact that our center is an allreferral-based NICU, with most of our ELBW patients being admitted after the first week of life. This made many of the typical outcome measures used in delivery hospitals inapplicable to our institution, but further highlighted the need for decreasing variability by having a unified approach to care specific to our EP population.

\section{Implementation}

\section{Development of a standard approach to care}

In 2004, with approval from hospital leadership, the process of developing the unified approach to EP infants took the form of drafting a set of guidelines for their care. It was clear from the outset that to be successful the caregivers involved must have a positive attitude and an investment in the EP infant. We chose to name this population "small baby" in part to have a positive and non-judgmental name for the population of EP infants to be served, thus we developed the SBG and eventually the small baby unit. In line with our overarching mission and vision, the SBG needed to not only focus on improving survival but also on parent engagement and optimizing developmental outcomes. Thus, the SBG addressed multidisciplinary parent-centered care and not just medication or weaning guidelines. A very important focus and purpose of the SBG was to serve as an agent for culture change.

A small baby committee consisting of representatives from all the disciplines involved in the care of the EP infant convened regularly to draft the SBG in a collaborative and cooperative fashion. The multidisciplinary team included physicians (with representatives from all three practices), neonatal nurse practitioners, nurses, respiratory therapists, pharmacists, nutritionists, physical therapists, occupational therapists, social workers, discharge planners, and psychologists. The small baby approach focused on an infantdriven model. In this model, the EP infant remained at the center of the care process and the emphasis was placed on humanizing the patient while stressing the role of the family in all aspects of care.

However, for most things there was no high-grade evidence available, therefore the focus was on decreasing variability in care. By working to achieve consensus across practices and across disciplines, we were able to deal with 
some of the more contentious issues including sedation while on mechanical ventilation, indomethacin prophylaxis, ventilator settings, ventilator weaning strategies, and indications for extubation. These patients require long lengths of stay due to "normal" developmental processes that must occur regardless of the underlying disease process prior to being ready for discharge. The focus of care changes as the EP patient achieves various developmental milestones during their NICU hospitalization. Therefore, we developed our SBG in three parts to allow for the appropriate developmental care that addressed the different needs of the EP infant as they achieved their milestones and moved toward discharge. Furthermore, the SBG dealt largely with aspects of care that may not be readily obvious to physicians, with the major focus on best practices related to bedside nursing care of EP infants. SBG1, covering the first week of life, was drafted, circulated widely for comment, and implemented in 2004. The guidelines incorporated available evidence-based practices and included ten subheadings: respiratory; skin; development; cardiovascular; patent ductus arteriosus (PDA); fluids/nutrition; neurological/sedation; laboratory studies; infection control; and family issues. We initiated the small baby program with the all-important first week of life as described for SBG1. As mentioned above, even though the majority of our EP infants are admitted after the first week of life, there is still a substantial number admitted after birth from smaller hospitals. It was important to have a foundation in place to cover this vital period of physiologic change, regardless of our admission statistics. After SBG1 was completely implemented, we moved to SBG2 which covers the second to fourth weeks of life, a transition from high illness severity to lower illness severity. Finally, after SBG2 was fully implemented we moved to SBG3, which covered the fifth week of life to discharge, a time usually of lower disease acuity with the major focus on attaining important developmental milestones. The team continued to focus on uniformity of care to optimize the likelihood of survival. Each "phase" of care required unique treatment challenges and opportunities for optimizing shortand long- term outcomes during these critical times of growth and development.

One foundational element in the implementation of the program has been the strong promotion of kangaroo care. The benefits of kangaroo care are vast and well defined in the literature [13], and the provision of kangaroo care has long been encouraged as soon as possible after birth, or in our case, after admission to the NICU [14]. It is our belief that the delivery of kangaroo care likely has a greater impact on the long-term health and outcomes of these children and families than any other intervention we routinely undertake on their behalf. It was considered imperative that kangaroo care be utilized as soon as possible in the first week of life. No infant was considered "too sick" to kangaroo care with the parent. To this day, this continues to be a foundation of our small baby program. The goal has been to minimize the time that the infant is in the isolette, and more recently this has been encouraged by having an adult-sized bed in the patient room. The parent is encouraged to hold the patient as long as possible, preferably while in the adult-sized bed. Routine care and minor procedures can be completed while the baby is in kangaroo care. A policy specific to care was established in conjunction with the SBG, understanding the detailed attention needed to minimize barriers to kangaroo care. With advances over the years in our electronic medical record, we have been better able to track and quantify how much kangaroo care our EP patients are receiving, which allows us to take further action to improve overall time in kangaroo care for the majority of the EP babies. We take pride in that we are able to continually find innovative ways to provide kangaroo care to even the most critically ill patients, regardless of the mode of respiratory support or other invasive devices.

Optimization of neurodevelopmental outcomes was a consistent focus in building and implementing each SBG. Part of this was obviously in the prevention of specific medical complications, such as trying to reduce rates of intraventricular hemorrhage by minimizing blood pressure fluctuations in the first week of life. A rather large focus, though, was in the specific developmental interventions by the medical team and family throughout the entirety of hospitalization, particularly kangaroo care and avoidance of noxious stimuli. The caregivers were reminded that all stimuli associated with medical care in a hospital could be potentially considered noxious to a small baby. Individualized support care that ensured the baby's self-regulation was incorporated in routine care and with procedures required during the hospitalization including but not limited to intravenous line placement, blood draws, daily weights, and blood pressure measurements. The child was to be handled minimally by the staff, while family interactions like kangaroo care were highly encouraged. This included routine assessments only every $6 \mathrm{~h}$, no routine weights for the first 3 days unless in-bed scales were used, and minimizing direct lighting (or shading of the baby's eyes). The common theme in our Small Baby Program has been to minimize painful stimuli while maximizing "joyful" experiences like kangaroo care, breastfeeding, and overall developmentally appropriate care. It should be clearly stated that the SBG are "living" documents and have now been revised many times as new potentially best-evidence becomes available for the care of the EP infant.

\section{Outcomes}

When first embarking on this mission of building and implementing a small baby program, our ultimate goal was 


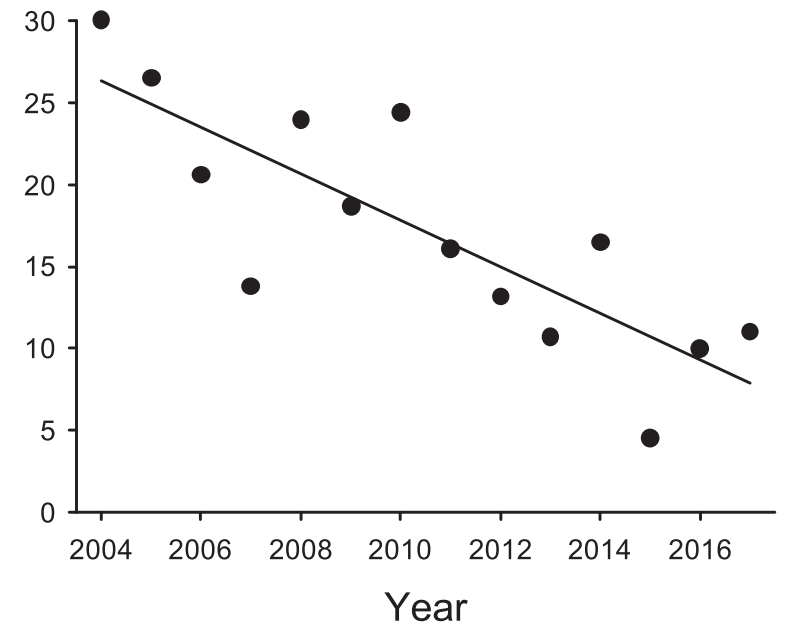

Fig. 1 Mortality of ELBW infants transferred to NCH from 2004 to 2017 decreased from $30 \%$ to $\sim \mathbf{1 0 \%}$. No reference cited as this is our own original figure.

to improve the care given to the EP infant. This is best objectively measured in the outcomes of these infants. While the program grew physically, so too did our efforts in tracking outcomes. The initial first step in this was to develop a small baby registry. Data has been entered on every patient admitted to the small baby program since its inception and has allowed us to not only publish our successes but has provided an avenue to continually refine our efforts towards those areas with the greatest challenges. As it pertains to outcomes, there is perhaps no better initial measure than a dramatic decline in our mortality rates of infants admitted to our small baby program from $30 \%$ in 2004 to nearly 10\% in 2017 (Fig. 1). One of our earliest reported successes as related to standardization of care was with improved fluid management and isolette humidity in the first week of life (Fig. 2A). Furthermore, we found that compared to the previous historical cohort, the group utilizing SBG had significantly greater survival without BPD, greater survival without severe IVH (Fig. 2B), and a reduced length of stay (Fig. 2C) [8].

Enhancing parental involvement has also been instrumental in improving outcomes. We continue to be extremely proactive in looking for ways to promote greater parental involvement and empowerment. This includes the use of kangaroo care, promoting the use of breastmilk and breastfeeding, and involving parents in the day-to-day care of their infant. In an effort to improve parental involvement in the small baby program we undertook a parent empowerment program and found that the parent empowerment program significantly reduced NICU length of stay while lowering readmission rates [15].

Other areas of focus have been the use of prophylactic indomethacin, de-escalating our approach to the PDA, and a proactive approach to early attempts at extubation from
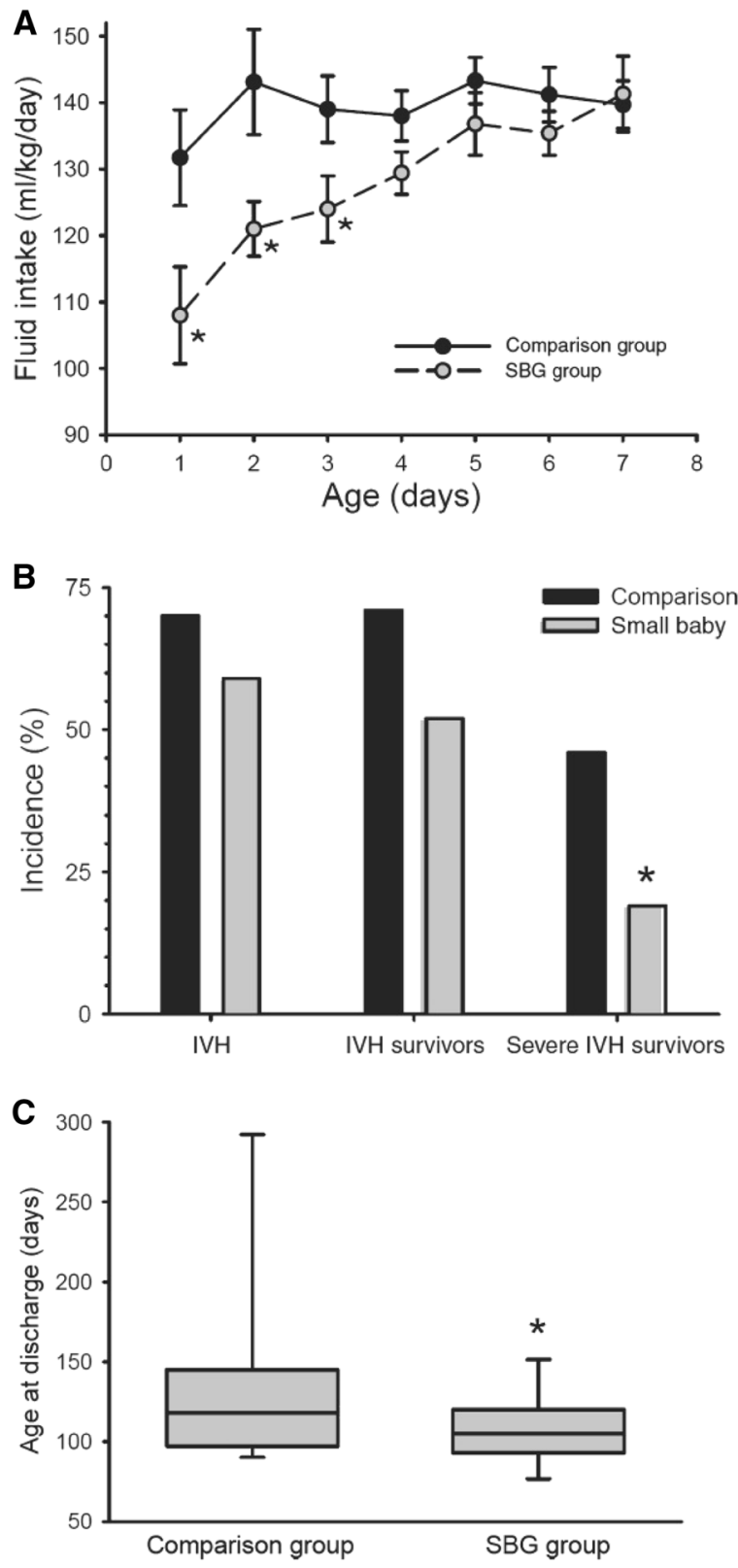

Fig. 2 Early successes of the Small Baby Program after implementing standardized guidelines. A Total fluid intake during the first $72 \mathrm{~h}$ of life was lower in the small baby group versus historical comparisons, $p<0.05$. Nankervis et al. [8]. Obtained by permission of Acta Paediatr. B Intraventricular hemorrhage in infants in small baby program versus comparison group, $p<0.02$. Severe IVH in survivors defined as grade III/IV in those who survived to discharge. Nankervis et al. [8]. Obtained by permission of Acta Paediatr. C Age of survivors at discharge significantly less in small baby program infants versus comparison group, $p<0.05$. Nankervis et al. [8]. Obtained by permission of Acta Paediatr.

mechanical ventilation. The use of prophylactic indomethacin was studied extensively in the 1990s, and the benefits seen at that time were thought to be from a decrease in rates of severe IVH and pharmacological closure of the PDA [16]. We included prophylactic indomethacin in SBG1. We have found that the use of 


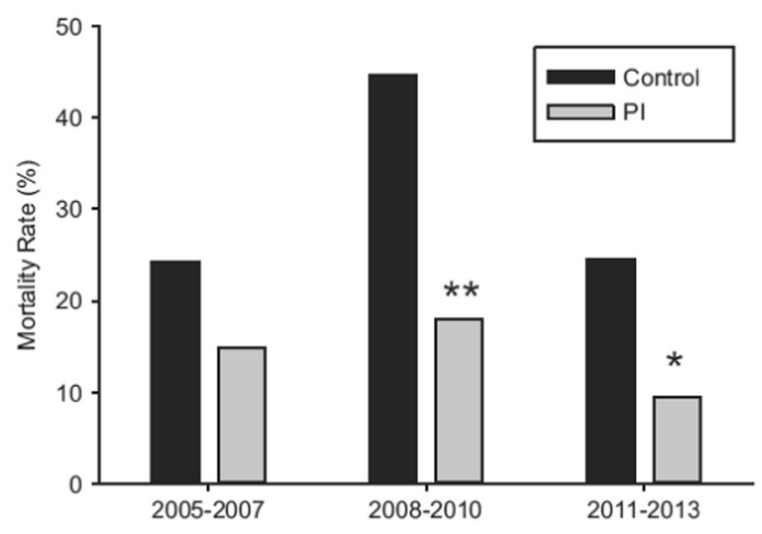

Fig. 3 Prophylactic indomethacin associated with reduced mortality in Small Baby Program patients during different time epochs. Use of PI not significantly different between epochs, and gestational age did not change over time $* p<0.05, * * p<0.005$. Nelin et al. [17]. Permission pending.

prophylactic indomethacin in babies admitted to the Small Baby Program was associated with a substantial improvement in survival (Fig. 3), with a relative risk reduction of $48 \%$ and a number needed to treat of only 7 [17]. Indeed, when we evaluate factors associated with mortality in patients admitted to the small baby program, the use of prophylactic indomethacin is one of the predictors of survival [18]. While we have not observed the previously reported effects of decreased rates of severe IVH, we have seen no other adverse events or worse neurodevelopmental outcomes during this time and as such, continue to promote the use of prophylactic indomethacin at birth in this specific population. The approach to the PDA has also evolved during this time period. In 2010 , our practice changed to significantly decrease the treatment of PDAs. In studying the EP infants admitted to the small baby program from 2008 to 2015, we found that decreasing the rates of PDA treatment was not associated with adverse effects on mortality or rates of BPD [19]. We also sought to change our culture and approach as it pertained to the initial extubation attempt. Historically, there was significant staff apprehension pertaining to the initial extubation attempt in an EP infant, with concerns regarding stability and the high likelihood of needing reintubation. This is obviously at odds with the knowledge that prolonged mechanical ventilation in this group is associated with the development of BPD. SBG1 strongly encouraged an extubation attempt to nasal CPAP early during the first week of life. We examined the association of the day of the first extubation attempt with the length of stay and the development of BPD. We found that in survivors the earlier the initial extubation attempt the shorter the length of stay and less need for supplemental oxygen, nCPAP, or mechanical ventilation at 36 weeks PMA despite the frequent need for reintubation [20].
Equally as important have been the strides we have made during this time in improving neurodevelopmental outcomes. The EP survivors from our unit have relatively low rates of cerebral palsy, with the majority having average Bayley scores as corrected for age at 18 months despite significant vulnerability related to extreme prematurity [21]. This continued trend of improved neurodevelopmental outcomes at 18-24 months has also been seen in the subsequent years since the implementation of the SBG. In fact, $80 \%$ of patients in this high-risk group of EP patients from our small baby program from 2008 to 2014 had cognitive scores $\geq 80$, $>70 \%$ had communicative scores $\geq 80$, and $>60 \%$ had motor scores $\geq 80$ on Bayley exams completed between 16 and 24 months (median 19 months). Even with an increase in survival during this time, the number of EP survivors with composite scores $<80$ did not increase [19]. A similar trend is highlighted when we evaluate BPD and neurodevelopmental outcomes, as the two have long been thought to be linked. Despite taking care of increasingly more cases of EP infants with varying degrees of BPD (and other co-morbidities) during this same time period, we have not seen any adverse effect with respect to neurodevelopment [22]. We attribute this again to the strong neurodevelopmental focus embedded within our SBG, regardless of the infant's primary underlying diagnosis. As with most of the co-morbidities we deal with, we strive to disconnect the immediate short-term pathophysiology from what we hope will ultimately be a more positive long-term outcome.

\section{Sustaining}

The journey towards sustaining and growing the small baby program at Nationwide Children's Hospital has required a multifaceted approach. Standardization of care across multiple disciplines, the establishment of guidelines, and parent-centered care have all played an important role in improving patient outcomes. No less important, however, have been the dedicated and heavily invested personnel that are the cornerstone of such an endeavor.

A core nursing team was established, consisting of nurses not only interested in the care of the extremely premature infant but also willing to set aside previous norms and agree to follow guidelines for consistency of care. This is vitally important when one considers the evidence with respect to protocol adherence. There is clear evidence that standardization of care and protocol adherence can dramatically improve morbidity and mortality specifically in the NICU setting [23, 24]. The concept of "decreased variability" is embedded into the SBG and in the various disciplines that form the foundation of the program. The core nursing staff for our small baby program currently consists of over sixty nurses and continues to grow. They have proven to be one of the cornerstones of the program, hailed 
for their versatility over the years as the SBG continues to evolve based on the latest evidence-based medicine. The small baby program has grown such that a full-time program coordinator is now needed to maintain the continued growth of the program through revisions of the SBG, quality improvement (QI) projects associated with implementing revisions to the $\mathrm{SBG}$, and continuous training of the small baby program physicians and staff. We have also developed a core group of attending neonatologists that work in the small baby unit and have agreed to follow the SBG; we as well have a dedicated medical director who has well-aligned clinical and research interests. Having dedicated staff with shared goals at all levels of the program has proven to be vital in its continued success and growth.

Since its inception in 2004, the small baby program has consistently maintained on average about 100 EP admissions per year, no small figure given their complex and often lengthy hospitalizations. With the growth of the program and its personnel over the years came another pressing need-physical space and the logistical questions that came with trying to build a unit ideally suited to both optimize neurodevelopmental practices while also promoting parentcentered care. The literature in this domain does support the use of single-family rooms when available, as benefits seem to include improved rest and healing, better infection/ environmental control, and are potentially associated with reduced mortality and shorter length of stay [25]. There is some evidence, however, that suggests the use of singlefamily rooms without family presence for prolonged periods of time may lead to sensory deprivation and worse developmental outcomes [26]. Our own data also suggests that those EP infants that lack prolonged human interaction seem to do worse on measurements that assess social competence and dysregulation [27]. The use of a "hybrid" system in which single-family rooms are used initially with the transition to an open bay model is gaining in popularity [28]. We emphasize though that based on the current literature, it is likely not the physical structure of the unit that is imperative for optimal development of the EP infant, but rather the need for prolonged human interaction when developmentally appropriate. Regardless of the type of physical structure or model used, "cohorting" our EP infants in a distinct location has allowed for greatly improved developmentally supportive care, adherence to protocols, and parent satisfaction. To complete the evolution of the Small Baby Program, the next planned step will be the implementation of a separate small baby NICU with its own physical space apart from the main NICU, clinical staff, and administration.

As the program has progressed, patients have become more complex. To address these complexities the multidisciplinary team meets weekly to perform "small baby rounds." These rounds are not meant to focus on the day-to- day care of the infant, but rather to address broader, systemic issues related to neurodevelopment, continuity of care, guideline adherence, parental support, and implementation and evaluation of on-going research or QI initiatives. We have found that building a core team of individuals focused on this very specific patient population has substantially changed our culture toward the EP infant and has affirmed a sense of accountability and reliability. This has helped to foster the identity of our program where individuals take great pride in the work they do and continually strive to improve outcomes, which then catalyzes and further sustains our small baby program via research, QI, or bedside teaching.

Sustaining our successes while still focusing on overcoming new challenges has taken many forms over the years; one area of continual focus has been improving upon our rates of bronchopulmonary dysplasia (BPD). This has recently taken the form of an on-going QI initiative called "Cure Me BPD," with specific aims targeting the reduction of oxygen usage at 28 days of life as well as our rates of any BPD and especially severe BPD at 36 weeks PMA. The high incidence of BPD in this population forced us to develop this specific QI project to "ramp up" our efforts in reducing variability in respiratory care. This initiative focused primarily on changing our unit culture with respect to the use of oxygen, better utilization of non-invasive ventilation, promoting early extubation attempts, and tackling systemic factors like nutrition and antibiotic stewardship. Since its inception in 2017, this initiative has allowed us to reduce our oxygen usage at 28 days of life from a baseline of $65-42 \%$. In addition, BPD rates have similarly decreased from $68 \%$ to $50 \%$.

As previously mentioned, an overarching goal for our small baby program has been to maximize "joyful" experiences for our patients while at the same time reducing or eliminating noxious stimuli. This is not done merely for the sake of patient comfort, but to optimize neurodevelopmental outcomes as there is evidence that cumulative noxious stimuli sustained by these patients can lead to worse outcomes [29]. One area of focus was on reducing unnecessary cuff blood pressure measurements in former ELBW premature infants close to being discharged home. These represent a classic example of noxious stimuli that in the vast majority of convalescing EP infants do not yield much clinical value. Using basic QI methodologies over a 1-year span, we were able to increase the number of eligible infants receiving appropriately fewer cuff blood pressure measurements from a baseline of $5 \%$ to over $70 \%$. Moving forward, we continue to strive to find a balance between reducing other noxious stimuli without impacting the care and outcomes of our patients.

The above examples highlight the importance of using both traditional research and QI to help ensure our practices 
Fig. 4 Small Baby Publications and Research Efforts. A brief list of publications generated from within our Small Baby Program and Small Baby Database.
- Nankervis CA, EM Martin, ML Crane, KS Samson, SE Welty, and LD Nelin. Implementation of a multidisciplinary guideline-driven approach to the care of the extremely premature infant improved hospital outcomes. Acta Paediatr. Feb;99(2): 188-193, 2010.

- Shepherd EG, TJ Calvert, EM Martin, JC Hitchner, SE Welty and LD Nelin. Outcomes of extremely premature infants admitted to a children's hospital depends on referring hospital. J Neonatal Perinatal Med 4(1): 45-53, 2011.

- Backes C, EM Martin and LD Nelin. A standardized approach to the care of the infant born at the threshold of viability. Neonatology Today 6(3): 1-7, 2011.

- Moorehead PA, EM Martin, EG Shepherd, NA Parikh, PB Reagan, W Gardner, KO Yeates and LD Nelin. Factors Associated with Survival of $<27$ Week Infants in an All-Referral Neonatal Intensive Care Unit. J Neonatal Perinatal Med 5(2): 105-111, 2012.

- Gonya J and LD Nelin. Factors associated with maternal visitation and participation in skin-to-skin care in all referral level IIIc NICU. Acta Paediatr. Feb;102(2):e53-6, 2013.

- Trittmann JK, LD Nelin and MA Klebanoff. Bronchopulmonary dysplasia and neurodevelopmental outcome in extremely preterm neonates. Eur J Pediatr. Sep;172(9): 1173-1180, 2013.

- Gonya J, E Martin, R McClead, L Nelin and E Shepherd. Empowerment programme for parents of extremely premature infants significantly reduced length of stay and readmission rates. Acta Paediatr. July;103(7):727-31, 2014

- Robbins ME, Trittmann JK, Martin EM, Reber KM, Nelin LD and EG Shepherd. Early extubation attempts are associated with reduced length of stay in extremely preterm infants even if re-intubation is necessary. J Neonatal Perinatal Med. 8(2):91-97, 2015.

- $\quad$ Bartman T, R Bapat, EM Martin, EG Shepherd, LD Nelin and KM Reber. Apgar score at 5 minutes is associated with mortality in extremely preterm infants even after transfer to an all referral NICU. Am J Perinatol. Nov;32(13):1268-72, 2015.

- Nelin TD, Pena E, Giacomazzi T, Lee S, Logan JW, Moallem M, Bapat R, Shepherd EG and Nelin LD. Outcomes following indomethacin prophylaxis in extremely preterm infants in an all-referral NICU. $J$ Perinatol. Aug:37(8): 932-937, 2017.

- Yossef L, EG Shepherd, S Lynch, KM Reber and LD Nelin. Factors associated with long-term mechanical ventilation in extremely preterm infants. J Neonatal Perinatal Med. 11(1): 29-35, 2018.

- Ezenwa B, Pena E, Schlegel A, Bapat R, Shepherd EG, Nelin LD. Effects of practice change on outcomes of extremely preterm infants with patent ductus arteriosus. Acta Pediatr Jan;108(1):88-93, 2019.

- Gonya J, Feldman K, Brown K, Stein M, Keim S, Boone K, Rumpf W, Ray W, Chawla N, Butter E. Human interaction in the NICU and its association with outcomes on the Brief Infant-Toddler Social and Emotional Assessment (BITSEA). Early Hum Dev. Dec;127: 6-14, 2018.

- Gonya J, Harrison T, Feldman K, Stein M, Chawla N. Nursing networks in the NICU and their association with maternal stress: A pilot study. J Nurs Manag. Mar;27(2): 442-449, 2019.

- Pishevar N, Fathi O, Backes CH, Shepherd EG, Nelin LD. Predicting survival in infants born at $<27$ weeks gestation admitted to an all referral neonatal intensive care unit: a pilot study. J Perinatol. May;40(5):750757,2020 are aligned with the best evidence-based care possible. Our program's culture of embracing innovation has allowed us to sustain the excellent work we have already achieved while ensuring a foundation exists to augment further change in the future. Figure 4 highlights additional areas of research and QI that have allowed us to improve our outcomes.

\section{Conclusion}

The care of the extremely preterm infant has evolved greatly since the initial inception of our small baby program in 2004. Early successes demonstrated by other countries such as Sweden and Japan have shown us how a change in staff attitude and culture greatly influences EP infant survival. At the core of this culture is a need for standardization and reduced variability in care. These basic tenets continue to drive the formation and implementation of all aspects of EP infant care in the small baby program at Nationwide Children's Hospital. We have found that this shared vision continues to attract members from different disciplines to our team. Throughout all of this, the inclusion of the parents in the journey to best outcomes is absolutely necessary. Together we continue to use traditional research and QI methodologies to promote and enhance the care of this patient population. The innovation to different approaches to care that have emerged from the small baby program has actually carried over to other populations within our NICU, leading to improved overall care for all of our patients. We also fully realize that there are many NICUs around the world that have remarkable outcomes without the use of a program or unit dedicated solely to the care of EP infants. Our experience is not meant to extol the potential benefits of a small baby program as an objectively superior approach to care. Nor do we suggest that a massive expenditure is required to bring about positive change, as our small baby program continues to focus on reduced variability, culture change, and not on additional spending. It is our sincerest hope that this description of our small baby program serves to capture our journey, challenges, and successes as we attempted to tackle a serious lack of care in this population in our institution and region. We understand that studying other "cultures-of-excellence" can lead to revaluating our own processes, and we continue to lean on this openminded approach to try to provide the best care possible. What was originally a patient population deemed to be too fragile to survive, has now shown all of us what true resiliency means.

Acknowledgements The authors would like to thank all of the providers from the various disciplines that have coalesced around a unifying goal of improving outcomes in our most vulnerable patients. From its inception to today, the growth and success of the Small Baby Program at Nationwide Children's Hospital would not be possible 
without such a selfless and collaborative effort. Furthermore, we want to recognize and thank all our patients and their families, from whom we learn every day.

\section{Compliance with ethical standards}

Conflict of interest OF, LDN, and EGS have no conflicts of interest to declare. KMR is a Board Member of the Children's Hospital Neonatal Consortium and receives financial compensation in this role.

Publisher's note Springer Nature remains neutral with regard to jurisdictional claims in published maps and institutional affiliations.

\section{References}

1. Norman M, et al. Association between year of birth and 1-year survival among extremely preterm infants in Sweden during 2004-2007 and 2014-2016. JAMA. 2019;321:1188-99.

2. Stoll BJ, et al. Neonatal outcomes of extremely preterm infants from the NICHD neonatal research network. Pediatrics. 2010;126:443-56.

3. Backes $\mathrm{CH}$, et al. A proactive approach to neonates born at 23 weeks of gestation. Obstet Gynecol. 2015;126:939-46.

4. Backes $\mathrm{CH}$, et al. Outcomes following a comprehensive versus a selective approach for infants born at 22 weeks of gestation. J Perinatol. 2019;39:39-47.

5. Brilli RJ, et al. The preventable harm index: an effective motivator to facilitate the drive to zero. J Pediatr. 2010;157:681-3.

6. Doerfler ME, et al. Methods for reducing sepsis mortality in emergency departments and inpatient units. Jt Comm J Qual Patient Saf. 2015;41:205-11.

7. James D, Pennardt AM. Trauma care principles. StatPearls. 2020. Treasure Island, FL.

8. Nankervis CA, et al. Implementation of a multidisciplinary guideline-driven approach to the care of the extremely premature infant improved hospital outcomes. Acta Paediatr. 2010;99: 188-93.

9. Shah PS, et al. Neonatal outcomes of very low birth weight and very preterm neonates: an international comparison. J Pediatr. 2016;177:144-52. e6

10. Smith LK, et al., An international comparison of death classification at 22 to 25 weeks' gestational age. Pediatrics, 2018;142.

11. Schneider K, et al. End-of-life decisions 20 years after EURONIC: neonatologists' self-reported practices, attitudes, and treatment choices in Germany, Switzerland, and Austria. J Pediatr. 2019;207:154-60.

12. Merritt TA, Gold M, Holland J. A critical evaluation of clinical practice guidelines in neonatal medicine: does their use improve quality and lower costs?. J Eval Clin Pract. 1999;5:169-77.
13. Boundy EO, et al. Kangaroo Mother Care and Neonatal Outcomes: A Meta-analysis. Pediatrics. 2016;137:e20152238. https:// doi.org/10.1542/peds.2015-2238.

14. Nyqvist KH. et al. Towards universal Kangaroo mother care: recommendations and report from the first European conference and seventh international workshop on Kangaroo mother care. Acta Paediatr. 2010;99:820-6.

15. Gonya J, et al. Empowerment programme for parents of extremely premature infants significantly reduced length of stay and readmission rates. Acta Paediatr. 2014;103:727-31.

16. Ment LR, et al. Low-dose indomethacin and prevention of intraventricular hemorrhage: a multicenter randomized trial. Pediatrics. 1994;93:543-50.

17. Nelin TD, et al. Outcomes following indomethacin prophylaxis in extremely preterm infants in an all-referral NICU. J Perinatol. 2017;37:932-7.

18. Pishevar N, et al. Predicting survival in infants born at $<27$ weeks gestation admitted to an all referral neonatal intensive care unit: a pilot study. J Perinatol. 2020;40:750-7.

19. Ezenwa B, et al. Effects of practice change on outcomes of extremely preterm infants with patent ductus arteriosus. Acta Paediatr. 2019;108:88-93.

20. Robbins M, et al. Early extubation attempts reduce length of stay in extremely preterm infants even if re-intubation is necessary. $\mathbf{J}$ Neonatal Perinat Med. 2015;8:91-7.

21. Moorehead PA, et al. Factors associated with survival of $<27$ week infants in an all-referral neonatal intensive care unit. J Neonatal Perinat Med. 2012;5:105-11.

22. Trittmann JK, Nelin LD, Klebanoff MA. Bronchopulmonary dysplasia and neurodevelopmental outcome in extremely preterm neonates. Eur J Pediatr. 2013;172:1173-80.

23. Coccia C, et al. Management of extremely low-birth-weight infants. Acta Paediatr Suppl. 1992;382:10-2.

24. Kastenberg ZJ, et al. Effect of deregionalized care on mortality in very low-birth-weight infants with necrotizing enterocolitis. JAMA Pediatr. 2015;169:26-32.

25. O'Callaghan N, Dee A, Philip RK. Evidence-based design for neonatal units: a systematic review. Matern Health Neonatol Perinatol. 2019;5:6.

26. Pineda RG, et al. Alterations in brain structure and neurodevelopmental outcome in preterm infants hospitalized in different neonatal intensive care unit environments. J Pediatr. 2014;164:52-60. e2

27. Gonya J, et al. Human interaction in the NICU and its association with outcomes on the brief infant-toddler social and emotional assessment (BITSEA). Early Hum Dev. 2018;127:6-14.

28. Erdei $\mathrm{C}$, et al. The growth and development unit. A proposed approach for enhancing infant neurodevelopment and familycentered care in the neonatal intensive care unit. J Perinatol. 2019;39:1684-7.

29. Cong X, et al. The impact of cumulative pain/stress on neurobehavioral development of preterm infants in the NICU. Early Hum Dev. 2017;108:9-16. 